\title{
Qualitative Study among African American Parents to Inform an Intervention to Promote Adoption of the Dietary Guidelines for Americans Food and Physical Activity Recommendations
}

\author{
Bernestine B. McGee, Valerie Richardson, Glenda S. Johnson, Crystal Johnson \\ Human Nutrition and Food Research Program, Southern University and A\&M College, Baton Rouge, \\ USA \\ Email: Bernestine mcgee@subr.edu
}

Received 9 January 2014; revised 9 February 2014; accepted 16 February 2014

Copyright (C) 2014 by authors and Scientific Research Publishing Inc.

This work is licensed under the Creative Commons Attribution International License (CC BY). http://creativecommons.org/licenses/by/4.0/

(c) $\underset{\mathrm{EY}}{\mathrm{B}}$ Open Access

\section{Abstract}

This qualitative study was conducted to enable the research team to culturally tailor an intervention to increase adherence to Dietary Guidelines for Americans (DGA) in African American parents and their children living in Lower Mississippi Delta (LMD) communities. Focus group results guided the planning of an obesity prevention intervention utilizing the We Can! (Ways to Enhance Children's Activity and Nutrition) obesity prevention program. Main outcome measure was perceptions of approaches to use in culturally tailoring a nutrition and physical activity intervention. Six focus group sessions were conducted with 86 African American adults to identify cultural concerns, intervention strategies in two Louisiana parishes in the LMD. Focus groups discussions were audio recorded, transcribed, and analyzed to identify recurring trends and patterns among focus groups. Major themes that emerged included cultural influences on food habits and physical activity, challenges of meeting the DGA and the CDC physical activity guidelines, facilitators and barriers to adhering to healthy food and physical activity guidelines and program development. Wider acceptance and use of the DGA recommendations are needed by LMD populations, leading to reduced prevalence of overweight and obesity and parallel reductions in the prevalence of chronic diseases. Interventions are needed that will enhance adherence to the DGA particularly for diverse subpopulations in the U.S. This study provides important insights for culturally tailoring an intervention to promote adoption of the DGA in two low-income African American Delta communities.

\section{Keywords}

African American Parents; Culture; Dietary Guidelines Adoption; Cultural Tailoring; Qualitative

How to cite this paper: McGee, B.B., et al. (2014) Qualitative Study among African American Parents to Inform an Intervention to Promote Adoption of the Dietary Guidelines for Americans Food and Physical Activity Recommendations. Food and Nutrition Sciences, 5, 835-849. http://dx.doi.org/10.4236/fns.2014.59093 


\section{Study; Knowledge and Perceptions}

\section{Introduction}

African Americans comprise a large segment of the Lower Mississippi Delta population and have the highest prevalence of obesity and its associated risks for chronic diseases [1]. The disparities pose a major challenge for policymakers and practitioners planning strategies for obesity prevention. Although there is science-based nutrition and health information available, it is not reflected in the African American community in dietary changes as reported by surveys [2] [3]. There is a need to identify effective strategies that will enhance adherence to the healthful food and physical activity recommendations set forth in the Dietary Guidelines for Americans (DGA) and the Physical Activity guidelines, particularly for diverse subpopulations in the United States.

An intricate relationship exists between food intake patterns and culture. Therefore, it is important to target specific demographic subgroups with tailored interventions to move all Americans toward achievement of the DGA [4]. Tailoring health education is a strategy used to bring individualization and personalization of health messages to members of a target group [5] [6]. Thus, targeted programs that deal with population-specific needs within a cultural group should also be tailored to the individuals within that group [7] [8].

The Social Cognitive Theory (SCT) is a sound theoretical framework for the development of an intervention addressing complex behaviors that act as barriers to adoption of healthful food and physical activity patterns [9]. SCT is based on the concept of reciprocal determinism, in which there is a dynamic interaction between environmental influences, personal influences, and behavior. SCT considers factors at both the individual and environmental levels to support behavioral change related to dietary intake and physical activity.

Focus group methodology has been used extensively to capture African Americans' perceptions of specific health conditions and various aspects of health behavior to implement culturally tailored or culturally sensitive interventions [6] [10]-[14]. Cultural sensitivity is defined by two dimensions: surface and deep structures. Surface structure involves matching intervention materials and messages to characteristics of a target population. This may involve using personal and environmental factors familiar to, and preferred by, the target audience. Surface structure refers to how well interventions fit within a specific culture. Deep structure involves incorporating the cultural, social, historical, environmental and psychological forces that influence the target health behavior in the proposed target population. Whereas, surface structure generally increases the "receptivity" or "acceptance" of messages, deep structure conveys salience [15].

Focus groups were used to guide the planning of an intervention to make it most acceptable to the participants and thus effective in increasing adherence to the DGA and the 2008 CDC Physical Activity Guidelines [16]-[18]. Focus group discussions with parents provided insight into cultural influences on dietary and physical activity patterns and overweight/obesity in an African-American population. Data derived from the formative evaluation will be used by the research team to determine intervention implementation strategies for designing an effective, culturally tailored nutrition/physical activity intervention for parents and their children.

\section{Material and Methods}

\subsection{Study Design}

Six focus group sessions were conducted in two Louisiana parishes for parents 18 years of age or older and six focus group sessions for children 8 to 13 years of age in the same parishes. Focus group sessions were conducted between January 2010 and January 2011 on different days of the week at local churches and parish Cooperative Extension offices. Only the findings from the focus groups sessions held with parents will be presented in this paper.

Recruitment of focus group participants was through outreach and direct appeal. Screening of participants was through a screening form which included demographic data. Participants were 18 years of age or older, a resident of the selected parish, responsible for preparing meals and purchasing food for the household, and parent of a child 8 - 13 years of age. Participants were served light refreshments and parent and child participants received a \$20.00 and \$10.00 gift card, respectively to encourage participation and compensate for time and travel expenses. Participants signed an informed consent form before each session. This study was approved by the 
Southern University Institutional Review Board.

Focus groups were conducted according to standard procedures [19]. Focus groups ranged in size from 8 - 10 individuals. The same focus group team conducted all six sessions. The duration of each focus group session was 75 - 90 minutes.

\subsection{Interview Questionnaire}

Questions were designed by the research team to determine perceptions of approaches to use in culturally tailoring an intervention designed to increase adherence to the DGA healthful food and physical activity recommendations. Content validity of the focus group question guide was established by a literature review of cultural tailoring indices and by consensus of the research team. Questions were informed by the constructs of and reciprocal interactions posited in the Social Cognitive Theory and the two dimensions of cultural sensitivity posited by Resincow [15]. Ten to 20 open-ended questions were developed. Questions were arranged from general to specific [19]. University faculty and staff reviewed questions for content and clarity and modifications were made based on their suggestions. Pilot focus group sessions were held to pretest the focus group questions and methodology with a similar group of parents and children.

Set I: Parent focus group questions

Questions were designed for parents to explore cultural experiences, history, beliefs, values and traditions that influence eating and physical activity behaviors and perceptions of health and obesity prevention (deep structure). Other influences explored included spirituality, social support and family pride and dedication. Participants responded to questions about weight status and challenges to meeting DGA and CDC physical activity recommendations and program development strategies for a healthy food choices and physical activity program (Table 1) [16]-[18].

Set II: We Can! Families Finding the Balance: A Parent Handbook focus group questions

We Can! is a national education program developed by the National Institutes of Health [20] and is designed for parents and caregivers to help youth ages 8 - 13 maintain a healthy weight. The program focuses on three

\section{Table 1. Parent focus group discussion questions.}

1) What does culture mean to you?

2) What are some cultural traditions or characteristics of Blacks in St. Landry Parish/East Carroll Parish?

3) What makes a person healthy?

4) What are two things that you believe or value that are very important to you?

5) Describe an attractive body size for women. B. Men. C. Children

6) Describe what you eat during a typical day during the week.

7) How does your culture influence your dietary habits?

8) How does your culture influence your physical activity habits?

9) What are some traditional foods or recipes that families eat in St. Landry/East Carroll Parish? B. Which of these foods would you consider healthy? Unhealthy?

10) Are you familiar with the USDA Dietary Guidelines for Americans? B. If so, what are some of these recommendations that people should follow to maintain health? I will highlight several of the key recommendations. How well do you meet the fruit/vegetable recommendation? B. How well do your children meet the fruit/vegetable recommendation? C. Are there challenges to meeting the fruit/vegetable recommendation? If so, what are they?

11) How well do you meet the fat recommendation? B. How well do your children meet the fat recommendation? C. Are there challenges to meeting the fat recommendation? If so, what are they?

12) Are you familiar with the 2008 Physical Activity Guidelines for Americans for adults and children? B. If so, what are some of these recommendations that people should follow to maintain health? I will read and highlight some of the key guidelines.

13) How well do you meet the 2008 Physical Activity Guidelines for Americans? B. Are there challenges to meeting the physical activity recommendation? If so, what are they?

14) What kind of physical activity do you enjoy?

15) What do you believe can be done to increase the amount of physical activity in your community?

16) On a scale of 1 to 5 , with 5 being the most confident, how confident are you that you could follow the Dietary Guidelines? B. Why did you provide that rating?

17) On a scale of 1 to 5 , with 5 being the most confident, how confident are you that you could follow the 2008 Physical Activity Guidelines for Americans? B. Why did you provide that rating?

18) Are there concerns about weight in adults in your community? B. In Children? C. If so, what are they?

19) Have you ever participated in a project, class or program which was designed to teach the participants about healthy eating? What did you like and dislike about it?

20) Suppose you were developing a program to promote healthy food choices and regular physical activity for parents and children in your community what topics should be included in this program? B. What activities should be included in this program? 
important behaviors: improved food choices, increased physical activity, and reduced screen time.

Parents reviewed the We Can! Families Finding the Balance: A Parent Handbook [20] for understanding and appeal. Questions captured participants' ideas and opinions for culturally tailoring the proposed intervention curriculum. Inquiry was made to determine whether materials were reflective of participants' language and cultural characteristics and delivery preferences of the intervention (surface structure). For example, participants were asked to respond to questions relative to organization/arrangement, print size, and font style of the information printed in the We Can! Handbook. Questions also addressed appeal and appropriateness of graphics, in particular, foods familiar to the intended audience, physical features and social network structures (family, friends, etc.) and settings (common time and place among the intended audience) of individuals portrayed. Questions also assessed participants' understanding of words, phrases and expressions used in the information (Table 2). Research has recommended assessment of these elements of educational materials for cultural sensitivity [21].

\subsection{Data Analysis}

Focus group data were analyzed following procedures recommended by Krueger [19]. At the conclusion of each session, a moderator/recorder report was completed which summarized key points, notable quotes, and overall findings. Audiotapes of all sessions were transcribed by a professional transcriber. Project staff listened to the focus group tapes and compared them against the written transcript. This enabled errors in understanding to be corrected. Using the transcripts, field notes, and moderator/recorder reports, the focus group team reviewed the data for the purpose of identifying recurring trends and patterns among the focus group sessions. This data were coded and sorted using the organizing framework of the discussion guide and the recurring trends and patterns. Topologies were constructed to determine if any data were omitted and agreement was reached relative to the organization of the data. The research team identified emerging themes from a list of most frequent responses by two or more focus groups and categorized according to SCT constructs. Each team member reviewed the summary of emerging themes to arrive at consensus for the final summarization. The most relevant quotes were included along with the emerging themes. A descriptive summary was compiled highlighting the most frequent and dominant responses. Prior to development of the final report to evaluate consistency, data, transcripts, field notes, and summary reports were reviewed and summarized by an independent reviewer.

\section{Results}

\subsection{Demographic Characteristics and Theme Identification}

A profile of the participants is presented in Table 3. The study population included 86 parent participants. Of the 86 participants, 70 (81\%) were age 18 - 44 years of age; 16 (19\%) were over 45 years of age. The majority of participants were female, 78 (91\%) and 8 (9\%) were male.

\section{Table 2. Focus group questions for We Can! Families finding the balance: A parent handbook.}

1) What were your feelings when you first picked the booklet up to read?

2) What are your thoughts about the front page and title of the booklet?

3) What are your thoughts on the pictures used in the booklet? Did they encourage you to read further? Are any changes needed?

4) What are your thoughts on the size and style of the letters used? Suggest changes if needed.

5) How would you describe the reading level of the booklet, for example, “difficult-to-read?” "not easy-to-read?” "hard-to-read?” "easy-to-read?" Was there a section that you found too technical or difficult or hard to understand? If so, which section? What changes are needed?

6) What topics were most interesting and useful to you?

7) What activities can be added to the lists related to physical activity (pp. 23-25) that are popular in your community?

8) Are there other foods which are frequently consumed in your community that are not listed or would make good examples: What other "go, slow" and "whoa" foods should be added to the lists? p. 16.

9) What are your thoughts on the section "What can my family and I do to encourage a healthy weight?” (pp. 4-6). Are the strategies suggested realistic for your family to follow? Explain your answer.

10) Are there other topics, information or thoughts that should be added to the booklet that would cause/encourage you to make needed changes in your eating and physical activity habits?

11) This fall, we plan to carry out a nutrition and physical activity campaign in the parish, focused on preventing overweight and obesity in children. What aspects of your culture should be included in the program that would make it special for you, and parents like you and therefore increase the likelihood of acceptance of the program? 
Table 3. Profile of focus group participants.

\begin{tabular}{lcc}
\hline \multicolumn{1}{c}{ Characteristic } & Number & $\%$ \\
\hline General focus groups & $\mathrm{N}=86$ & \\
Parents & & \\
Age & & \\
$18-44$ & 70 & 81 \\
$45+$ & 16 & 19 \\
Gender & 78 & 91 \\
$\quad$ Female & 8 & 9 \\
$\quad$ Male & $\mathrm{N}=18$ & 89 \\
We Can! focus groups & 16 & 11 \\
Female & 2 & \\
Male & & \\
\hline
\end{tabular}

This study is subject to some limitations. The results of the study may only be relevant for an African American population. The sample was not randomly selected; however, recruitment information was widely distributed through different venues in the two parishes. Participants were majority female, likely a result of the selection criterion that specified that the participant be the primary food purchaser and preparer. The intervention strategies proposed may not be applicable to men.

\subsection{SCT Constructs and Cultural Sensitivity Themes}

Major themes were categorized by the SCT constructs and by the cultural sensitivity dimensions, deep structure and surface structure based on the most frequent responses by three or more focus groups. Table 4 summarizes the SCT themes identified, personal and environmental determinants.

\subsubsection{Personal Determinants}

Personal determinants derived from the data included knowledge and skills and body size perceptions. Environmental determinants included challenges of meeting the DGA and the CDC physical activity guidelines, and facilitators and barriers to adhering to healthy food choices and physical activity guidelines. The deep structure themes that emerged were cultural influences on food habits and physical activity and the surface structure theme identified was program development.

Table 4 summarizes responses to inquiry about the Dietary Guidelines for Americans. The groups were not very familiar with the DGA. Approximately 50\% of the participants indicated that they meet the fruit but not the vegetable recommendation. Few groups indicated that children meet the recommendation. The school lunch program was cited as helping children to meet the fruit and vegetable recommendation. Participants were not familiar with the 2008 Physical Activity Guidelines for Americans and did not participate in the recommended levels of physical activity. However, all groups indicated that they meet the guideline through the kind of work they were performing and from taking care of children.

All focus groups felt that there were concerns about weight in adults in the community. The related health concerns mentioned were high blood pressure, diabetes, asthma and heart disease. There was less concern about weight in children in the community.

Participants described an attractive body size for women relative to dress size ranging from size 18, 14, and 12, respectively. Most of the participants described an attractive body size for men relative to physique. An attractive body size for children was based on the individual child.

\subsubsection{Environmental Determinants}

Participants indicated that quality, availability and cost of fruits and vegetables were obstacles in meeting DGA for fruits and vegetables. All focus groups indicated that they as well as their children do not meet the DGA fat 
Table 4. Social cognitive theory constructs.

Constructs

Personal determinants

Body size perceptions

Describe an attractive body size for:

A. Women

Size 12

Size 14

Size 18

B. Men

Tall; muscles; built; slender built

C. Children

Depends on the age and size of the child

Not being over or under weight

Knowledge and skills

Perception of healthy

Eating right/healthy

Exercising

Resting/sleeping

Watching your portions

Dietary guidelines for American

Familiar with DGA

Unfamiliar with DGA

Most familiar with the fruit and vegetable recommendation

Adults meet the fruit rather than the vegetable recommendation

Children do not meet the fruit and vegetable recommendation

Fat recommendation not met

Challenges to meeting the physical activity guidelines

Busy/lack of time

Weather; available safe facility

Confidence scale for following the DGA

Five

Four

Three

Two

Confidence scale for following the physical activity guidelines

Four

Three

Two

Environmental determinants

Challenges to meeting the fruit and vegetable DGA recommendations

Too expensive; quality and quantity of fruits and vegetables 
recommendation. Failure to meet the fat recommendation was attributed to the consumption of high calorie foods and snack foods.

Challenges to meeting the physical activity guidelines included lack of time, safety, tired from work, weather, and no facility in the community for working out. Walking was the preferred activity indicated by a majority of the participants followed by riding bikes.

Based on a scale of 1 - 5, focus group participants indicated a moderate degree of confidence that they could follow the DGA (Table 4).

\subsubsection{Deep Structure}

Cultural influences on food habits and physical activity. Table 4 summarizes the responses to five questions intended to gain participants' perspective on the concept of culture and mediating factors that could impact lifestyle change toward improved food habits and increased physical activity. Participants perceived God, family, health, education, and character as important to them. Family traditions such as holiday celebrations, family meals, and cooking methods influence eating habits. Physical activity habits were influenced by patterns of parents, while approximately a third of the groups indicated that neighborhood safety and busy schedules (Table 5).

The traditional foods or recipes most frequently mentioned by $37 \%$ of groups were greens and fried chicken; gumbo; potato salad; barbeque; boiled crawfish; sweet potato pie; boudin; jambalaya; turkey necks; and homemade cake. When asked which traditional foods are considered healthy, participants responded that it depends on how it is prepared.

\subsubsection{Surface Structure}

Program development. Table 5 presents the program development perceptions of participants. Varied responses were provided relative to previous participation in healthy eating educational activities, but the most common responses were weight watchers, church weight loss challenge, cooperative extension service nutrition programs and through place of employment. Programs most preferred by participants were menu modification, recipes, and food demonstrations.

To increase the amount of physical activity in the community, two-thirds of the groups indicated having access to facilities in the community such as a park, recreation center; group physical activity; and support groups. Participants also suggested organized sports for children and recess in school and provision of playground equipment.

When asked what topics should be included in a program to promote healthy food choices and regular physical activity for parents and children in your community, participants indicated food demonstrations, interactive family oriented nutrition and exercise activities and provide incentives (food, prizes, money). Of approaches mentioned by participants to include in a program to promote regular physical activity for parents and children, access to safe facilities was most frequently cited followed by group activities; after-school health programs; and safe places for activities.

Table 6 summarizes participants' perspective following a review of We Can! Families Finding the Balance: A Parent Handbook for understanding of the text and appeal. All four groups indicated that the handbook was colorful and eye catching and depicted family togetherness and families eating and exercising together. Participants indicated that the printing on the cover should be in black rather than white. Participants indicated that there should be more Black families on the cover. In the body of the handbook, a majority of the groups indicated that there should be more pictures of Blacks and pictures of local scenes. No changes were suggested for size and style of the font used. All four groups indicated that the Handbook was "easy-to-read".

Participants indicated that the section on Calorie Count and BMI information was technical and should include more examples. The most interesting topics indicated by participants were statistics; Energy In; Energy Out; Go (eat almost anytime), Slow (eat sometimes, or less often), and WHOA (eat only once in a while or on special occasions) foods; energy requirements; and portion size information. Participants suggested that other activities should be added to the physical activity list including interactive video games (Wii), Zydeco dancing; rollerblading, skateboarding, skating, baseball, and tennis.

Participants suggested the addition of foods consumed in the local area to the list of "GO, SLOW and WHOA foods". Suggested foods included: "GO" foods: wild game (rabbit, deer), goat, soymilk, yams, salad, healthy snacks; powerades; "SLOW" foods: fried chicken; and "WHOA" foods: boudin, cracklings, chitterlings, neck bones, wild game (deer, rabbit), gumbo, alcoholic beverages, and pickles. 
Table 5. Cultural sensitivity dimensions.

\section{Deep structures}

Cultural values/traditions

Definition of culture

Background

Traditions; way of life

Family; heritage; God/spirituality

Important values

Family

Health

God; education; character

Cultural traditions

Food

Family gatherings

Church gatherings; Zydeco; festivals

Cultural influence on food habits

Brought up eating fried food, boudin, cracklings

$\begin{array}{ll}\text { Grew up eating vegetables } & 50\end{array}$

Family cookouts; eating wild game; cooking techniques 37

Health crisis

Cultural influence on physical activity habits

Parents were more active, farming

Neighborhood safety; busy schedules

\section{Surface structures}

Suggestions for increasing physical activity in the community

Facilities

Support groups

Organized sports for children; playground equipment;

Recess in school

Preferred physical activity

Walking and bicycle riding

Healthy food choices program development suggestions

Healthy food demonstrations and interactive lessons $\quad 50$

$\begin{array}{ll}\text { Provide incentives (food, prizes, money) } & 37\end{array}$

Healthy food cook-off; gardening $\quad 37$

Physical activity program development suggestions

Group physical activity $\quad 55$

Variety of sports; afterschool program activities 50 
Table 6. Summary of review of We Can! Families finding the balance: A parent handbook.

\begin{tabular}{cc}
\hline Dimension & Sample responses \\
\hline Surface structure &
\end{tabular}

1) What were your feelings when you first picked the booklet up to read?

2) What are your thoughts about the front page and title of the booklet?

3) What are your thoughts on the pictures used in the booklet? Did they encourage you to read further? Are any changes needed?

4) What are your thoughts on the size and style of the letters used? Suggest changes if needed.

5) How would you describe the reading level of the booklet, for example, "difficult-toread?" "not easy-to-read?" "hard-to-read?" "easy-toread?" Was there a section that you found too technical or difficult or hard to understand? If so, which section? What changes are needed?

\section{Deep structure}

6) What topics were most interesting and useful to you?
Colorful; family togetherness; family eating healthy and exercising; Family eating together and doing physical activity with kids; It's not too thick; I thought, we got some black people on the book.

Shows a lot of families, shows families exercising; It shows families together; Positive outlook; Encouraging, the title encourage what we can do; Eye-catching; Good title; We Can, so the title tells us if we all try hard enough, we really can; What is the President's slogan, Yes, we can-if the President is saying we can do it, we can do it. Decrease the amount of blue background; Enlarge the picture, the blue covers more than the picture; Graphics in the middle should be larger, the blue will throw you off;

title needs to be at the top of the book; be bolder;

Write in black instead of white.

Appropriate, no changes needed (all agreed); to me they did a good job, a very good job. Show families it's a good idea to spend time together, get away from T.V. and video games; Parents preparing healthy meals; ....so they encouraged their kids to be involved in eating healthy foods.

Colorful; food is colorful and looks like it's just waiting for you to just eat it off the plate; Good choices because we as Black Americans, we need to promote good

eating habits and physical activity and diet and parents eating together with their children and spending time;

Shows different activities they could do. If someone picked up the book and couldn't read, they could look and see them riding a bicycle or playing ball or whatever without reading; Changes:

Comparison of good and bad... show unhealthy and come back with healthy;

Pictures of children in local activities (riding horses);

More pictures of black families eating together and doing physical activity with kids;

More pictures of minorities; more pictures of families from the parish;

Families working in gardens;

Celebrity pictures;

Pictures of yams, cornfields.

"Appropriate;" "very good"

"No changes to font; you can read with or without glasses; good, anyone can read it;"

"I like where the title was done in color and the information was done in another color"

I think a $6^{\text {th }}$ grader can read it; it was very understanding; and easy to read and to the point; not to technical; simple and basic, as far as the pyramid and everything broken down; I like the way they left the website so you could go to the website and get other information

Downside of overweight;

Energy balance;

Handy guide to calories burned in common activities;

Encourage healthy weight;

BMI information;

Parents as role models;

The calorie requirements;

Portion size information;

I thought the part was interesting where the family can help encourage a healthy weight;

Nutrition label information...that might encourage parents to be conscious of looking at the labels and reading them more when they're purchasing; I like them all. 


\section{Continued}

7) What activities can be added to the lists related to physical activity (pp. 23-25) that are popular in your community?

8) Are there other foods which are frequently consumed in your community that are not listed or would make good examples: What other "go, slow" and "WHOA" foods should be added to the lists? p. 16.

9) What are your thoughts on the section "What Can My Family and I Do to Encourage a Healthy Weight?” (p. 4-6). Are the strategies suggested realistic for your family to follow? Explain your answer.

10) Are there other topics, information or thoughts that should be added to the booklet that would cause/encourage you to make needed changes in your eating and physical activity habits?

11) This fall, we plan to carry out a nutrition and physical activity campaign in the parish, focused on preventing overweight and obesity in children. What aspects of your culture should be included in the program that would make it special for you, and parents like you and therefore increase the likelihood of acceptance of the program?
Basketball, baseball, rollerblading, skateboarding, video game exercises, mowing the lawn, skating, Zydeco dance.

GO foods: beverages, Salad, healthy snacks, powerades, greens, fruit salad, wild game (deer, rabbit), soy milk, yams;

SLOW foods: fried chicken;

WHOA foods: alcoholic beverages, pickles, chitterlings, tripe, neckbones, gumbo, barbeque, boudin, cracklings, wild game (deer, rabbit), bread.

No, it's hard; we can't afford to eat healthy all the time; paint a more realistic picture, high cost of eating healthy, compare unhealthy to healthy and long life.

Yes, it's realistic.

To exercise more and make healthy food choices; good, because it encourages eating together and healthy eating.

More exercise; healthy food choices.

Convert family plan to individual;

Focus on health and not the size of a person;

Cater to healthier food options at fast food places;

Consequences (statistics) of eating healthy;

Information on budgeting for healthy meals; healthy snacks;

Include support group (counselor/motivator) and contact numbers;

Written well, encouraging everyone to get a fresh start;

Covered just about everything good;

Start to changing our eating style;

Good eye opener to a lot of people;

Help us better understand versus some of the downfalls of not eating healthy;

Colorful plates;

Food pyramid.

\section{Group sessions}

Form walking groups; aerobics

Early childhood programs

Involve school system/board

Flyers; let parents know the importance with flyers

Recreational park/fitness center

Music; Zydeco; dancing

Incentives; t-shirts, pedometers

Accountability

Testimonials; use community peers to give testimonials

Fun/family activities

Community event/ activities

Bike-a-thon; hay ride, fun day in park;

Use local professional athletes, doctors

Got to have something to eat and entertainment

Have more details about the consequences of obesity

How do we get more men involved? have them compete with each

other; gym...lift weights

When asked to respond to the chapter in the handbook, "What can my family and I do to encourage a healthy weight", participants felt that the strategies should include a sample family plan. A related quote was "family plan with kids, give an incentive for keeping up with plan”. The groups were divided regarding whether the strategies were realistic. Half of the group indicated that it would be a challenge to follow the recommendations.

In response to the inquiry about local cultural aspects to include in a program focused on preventing overweight and obesity in children, participants indicated participant accountability, family and community activities; marketing by local professional athletes and doctors; music; dancing/Zydeco; form walking groups; provide aerobics; offer incentives; involve school board for early childhood intervention and provide more details about the consequences of obesity. Participants indicated that it was important to get more men involved in the program. 


\section{Discussion}

Understanding the dynamic interaction between the environment, a person's behavior, personal factors and cultural values is important for facilitating the adoption of the DGA. There are gaps in our understanding of effective strategies that will increase adherence to the DGA particularly for diverse subpopulations in the US. Through this study important themes were identified to inform an intervention to promote adoption of the DGA among African American parents in two Lower Mississippi Delta communities. The qualitative findings have four major results: 1) facilitators and challenges to adhering to healthy food choices and physical activity guidelines, 2) challenges of meeting the DGA and the CDC physical activity guidelines, 3) identification of cultural influences on food habits and physical activity, and 4) identification of strategies for cultural tailoring curriculum content.

Considerable variability was found in the perceptions of factors influencing healthy food consumption behavior in Delta communities, including health concerns, family influence, and need for and availability of nutrition information [22]. Research suggests that lifestyle behaviors of African American females are responses to historical, social, and cultural forces affecting their personal health beliefs, attitudes, and perceptions [23]. Though unhealthy diet and physical inactivity are clearly determinants of excess weight in this group, few data exist on the psychosocial factors (beliefs, attitudes, and perceptions) that promote these behaviors and the cultural contexts within which they operate [24]. In this study, focus group discussions uncovered beliefs, attitudes, and ideas about diet, physical activity, and health that relate to adoption of the DGA. Similar to previous research, participants displayed a superficial understanding of the meaning of health and the relationship of food choices and physical activity [25]. Participants showed a pattern of consumption of foods high in fat, sugar and sodium; eating fast food; unplanned snacks; and overeating along with low levels of physical activity. Findings from our study are largely consistent with existing studies [23]-[25].

Participants displayed a preference for a larger body size. Studies show African Americans tend to accept larger body sizes. This tolerance of larger sizes has lead to an increase in obesity and more obesity/health related illness [26]-[28].

Consistent with existing studies which characterized the importance of core cultural values in African Americans, this population perceived God, family, health, education, character, and cultural traditions as important. Focus group discussions revealed variability in perception of factors that influence eating and physical activity behavior. Lifestyle behaviors are a response to historical, social and cultural influences. The family is widely recognized as being significant in food decisions. Research shows the shaping of food choices takes place in the home. Because family and friends can be a source of encouragement in making and sustaining dietary change, adopting dietary strategies which are acceptable can benefit the individual and have an effect on the eating habits of others [29].

Eating and physical activity patterns that are focused on consuming fewer calories, making informed food choices, and being physically active can help people attain and maintain a healthy weight, reduce their risk of chronic disease, and promote overall health. Scientific evidence suggests that the dietary and physical activity recommendations described in the DGA may help people attain and maintain a healthy weight, reduce the risk of chronic disease, and promote overall health. The DGA accommodate the varied food preferences, cultural traditions, and customs of the many and diverse groups who live in the United States [18]. Although some participants indicated that they were not familiar with the DGA, they were aware of the need to eat more fruits and vegetables. Participants cited challenges of cost and availability. Research has shown that among low income individuals who think they should eat more fruits and vegetables, indicated that inadequate access to quality and affordable fruits and vegetables in their home, school, work, and community environments were barriers to eating more fruits and vegetables [30]. Limited access to healthy and inexpensive foods in low income communities is a major barrier for obesity prevention and good health. Studies have shown that there are fewer grocerystores with affordable fruits and vegetables, and other low-fat items close to low-income neighborhoods and in rural communities [31]-[33].

Participants in this study were not aware of the CDC Physical Activity Guidelines and were not meeting the guidelines for adults. Consistent with previous research, this study found that this population participates in less physical activity and similar barriers were cited such as lack of motivation, safety issues, weather, limited access to physical fitness programs and facilities, and lack of social support. African Americans are less likely to meet recommended levels of physical activity, a lifestyle factor critical to altering chronic disease risks. Such high 
levels of insufficient physical activity underscore a need for better dissemination and adoption of strategies to increase physical activity levels within these high-risk communities [34] [35]. A review to examine consumer response to healthy eating, physical activity and weight-related guidelines on behavior found that many respondents reported that the guidelines were confusing, and that simple, clear, specific, realistic, and in some cases, tailored guidelines are required. Recognition of guidelines did not signify understanding nor did perceived credibility of a source guarantee utilization of guidelines [36].

There was concurrence on the opportunities suggested by participants for meeting the guidelines and those recommended by the CDC. According to the Centers for Disease Control and Prevention's (CDC's) Guide to Community Preventive Services [37], the evidence-based strategies to increase physical activity levels include the following: community-wide campaigns, individual behavioral change, social support in community settings, the creation or enhancement of access to places for physical activity, and point-of-decision prompts.

Culture, defined as the unique shared values, beliefs, and practices of a group, can influence the behaviors of individuals by affecting their thoughts, feelings, acceptance, and adoption of health education messages [38] [39]. For example, to reduce the prevalence of excess weight, current health strategies promote lower fat intake. However, among African American populations, especially in the southeastern United States, consumption of foods high in fat is a documented dietary and cultural practice [40]. This study found that cultural factors affect food choices, physical activity levels and body size satisfaction. Findings from our study are largely consistent with existing studies. Adults in the LMD consume $20 \%$ fewer servings of fruits and vegetables compared to national intakes and have higher intakes of fat and lower intakes of several micronutrients [41]. In African-American culture, food has been the center of social interaction. Research found that African-Americans in north central Florida felt that eating healthier food meant giving up part of their culture. Sunday dinner composed of family favorite foods is an integral part of African American culture [42].

The diets of most Americans fall short of the Dietary Guidelines for Americans [43]. The participants in study were not overwhelmingly confident that they could adhere to the DGA or the physical activity guidelines. Participants indicated that the support of family and friends was important for changing behavior. This highlights the need for healthy lifestyle interventions to be tailored to the specific population with consideration given to inclusion of support groups and family-centered activities. Healthy eating and physical activity strategies should focus on reducing intakes of high-calorie, low nutrient foods such as sugar-sweetened beverages, high-fat dairy, and desserts while promoting as alternatives water, fat-free or low-fat milk, and fruits or low-fat/low calorie dessert choices [44]. In community settings, the creation or enhancement of access to places for physical activity is important [37].

Health promotion interventions will be more effective when they are culturally appropriate for the population served [8]. Understanding personal and external determinants of food choices and physical activity and application of deep and surface structure elements for modifying food and physical activity behavior are necessary to plan effective, culturally sensitive, and relevant interventions aimed at increasing adoption of healthy food and physical activity recommendations. The surface structure dimensions identified by this LMD population included a variety of interactive program strategies and barriers to promoting healthy food choices and regular physical activity. Surface structure elements identified include language, facilities, location, music, food and nutrition messages. Research has shown that there is limited or no access to physical fitness programs and facilities in low-income communities [43]. Similarly, previous research has found that participants want a variety of active learning intervention strategies [44]-[47]. New culturally relevant, credible, and trusted approaches for health promotion must be identified for health status indicators to improve for African American populations. These data therefore suggest a potential role for cultural tailoring to enhance the cultural appropriateness and, ultimately, program outcomes, of healthy eating and physical activity interventions.

Overall, focus group participants approved of the We Can! Families Finding the Balance Handbook. The Parent Handbook received the 2005 NIH Plain Language Award for an Outstanding Plain Language Product [23]. Some modifications to the Handbook were recommended including ethnic pictures, local foods, familiar physical activities and other topics for maintaining healthy weight, nutrition, and physical activity including healthy cooking and budgeting. Local cultural aspects were cited as important to energize participants and better equip them to maintain a healthy lifestyle. Deep structure dimensions identified suggest that culture, history, religion, friends and family and environment play a role and influence food choices and physical activity behavior.

Local cultural aspects were cited as important to energize participants and better equip them to maintain a healthy lifestyle. Deep structure dimensions identified suggest that culture, history, religion, friends and family 
and environment play a role and influence food choices and physical activity behavior.

\section{Conclusion}

More effective health promotion programs to reach African American parents and their children living in LMD communities are needed to increase adherence to the healthful food and physical activity patterns set forth in the DGA. Qualitative research methods to develop health promotion programs that are culturally targeted to the issues common to the population being served and tailored to the needs and interests of the population may be an effective approach to increase adoption of the healthful food and physical activity guidelines. Focus groups gave important insights into likelihood of this LMD population adopting the DGA. Focus groups data is important for designing interventions strategies that can appropriately target needs within the LMD. The results of this study instructed us to emphasize a holistic concept of health that includes high self-esteem and achievement orientation, value systems and norms, spirituality and cultural awareness as well as physical health, role of food, a community atmosphere and social support, and involving extended family members. It is anticipated that appropriately adapting Dietary Guidelines to culture, economic and social differences will result in reduced weight gain and improved health risks in African American parents and their children living in Delta communities.

\section{Acknowledgements}

The project described was supported by the Agricultural Research Service, United States Department of Agriculture, Project No 58625118041. The content is solely the responsibility of the authors and does not necessarily represent the official views of the Agricultural Research Service.

\section{Authors' Contributions}

BBM conceptualized and drafted the paper. Each author contributed to further development and revisions of the paper and assumed a unique role in execution of the research. All authors read and approved the final manuscript.

\section{References}

[1] Lower Mississippi Delta Nutrition Intervention Research Consortium (2004) Self Reported Health of Residents of the Lower Mississippi Delta. Journal of Health Care for the Poor and Underserved, 15, 645-662. http://dx.doi.org/10.1353/hpu.2004.0056

[2] Bronner, Y.L., Harris, E., Ebede, T.L., Hossain, M.B. and Nowverl, A. (2001) Historical Assessment of Nutrition Studies Using Only African-American Study Subjects: Gender, Socioeconomic Status, and Geographic Location. Ethnicity and Disease, 11, 134-143.

[3] Bokat, R. (2006) The Failure of the Dietary Guidelines to Promote the Health of a Culturally Diverse America: An Abandonment of Social Science Principles Challenging Dogma. Challenging Dogma On-Line Blog. http://sb721.blogspot.com/2006/12/failure-of-dietary-guidelines-to.html

[4] Stables, G.J., Subar, A.F. and Patterson, B.H. (2002) Changes in Vegetable and Fruit Consumption and Awareness among US Adults: Results of the 1991 and 1997 5-a-Day for Better Health Program Surveys. Journal of the American Dietetic Association, 102, 809-817. http://dx.doi.org/10.1016/S0002-8223(02)90181-1

[5] Campbell, M. and Quintiliani, L. (2006) Tailored Interventions in Public Health: Where Does Tailoring Fit in Interventions to Reduce Health Disparities. American Behavioral Scientist, 49, 775-793.

[6] Strolla, L.O., Gans, K.M. and Risica, P.M. (2006) Using Qualitative and Quantitative Formative Research to Develop Tailored Nutrition Intervention Materials for a Diverse Low-Income Audience. Health Education Research, 21, 465-476. http://dx.doi.org/10.1093/her/cyh072

[7] Kreuter, M.W., Bull, F.C., Clark, E.M. and Oswald, D.L. (1999) Understanding How People Process Health Information: A Comparison of Tailored and Non-Tailored Weight-Loss Materials. Journal of Health Psychology, 18, 487-494. http://dx.doi.org/10.1037/0278-6133.18.5.487

[8] Kumanyika, S.K., Whitt-Glover, M.C., Gary, T.L., Prewitt, E., Odoms, A.M., Banks-Wallace, J., Beech, B.M., Halbert, C., Karanja, N., Lancaster, K.J. and Samuel-Hodge, C. (2007) Expanding the Obesity Research Paradigm to Reach African American Communities. Preventing Chronic Disease, 4, 456-471.

[9] Bandura, A. (1977) Self-Efficacy: Toward a Unifying Theory of Behavioral Change. Psychological Review, 84, 91-215. http://dx.doi.org/10.1037/0033-295X.84.2.191 
[10] Dannelly, J.M., Kicklighter, J.R. and Hopkins, B.L. (2005) Recommendations for Nutrition Interventions with Overweight African-American Adolescents and Young Adults at the Atlanta Job Corps Center. Journal of Health Care for the Poor and Underserved, 16, 111-126. http://dx.doi.org/10.1353/hpu.2005.0008

[11] Dietz, W.H. and Gortmaker, S.L. (2001) Preventing Obesity in Children and Adolescents. Annual Review of Public Health, 22, 337-353. http://dx.doi.org/10.1146/annurev.publhealth.22.1.337

[12] Kannan, S., Webster, D., Sparks, S., Acker, C.M., Greene-Moton, E., Tropiano, E. and Turner, T. (2009) Using a Cultural Framework to Assess the Nutrition Influences in Relation to Birth Outcomes among African American Women of Childbearing Age: Application of the PEN-3 Theoretical Model. Health Promotion Practice, 10, 349-358. http://dx.doi.org/10.1177/1524839907301406

[13] Burnet, D.L., Plaut, A.J., Ossowski, K., Ahmad, A., Quinn, M.T., Radovick, S., Gorawara-Bhat, R. and Chin, M.H. (2008) Community and Family Perspectives on Addressing Overweight in Urban, African-American Youth. Journal of General Internal Medicine, 23, 175-179. http://dx.doi.org/10.1007/s11606-007-0469-9

[14] Horodynski, M.A. and Arndt, M.J. (2005) "Eating-Together” Mealtimes with African-American Fathers and Their Toddlers. Applied Nursing Research, 18, 106-109. http://dx.doi.org/10.1016/j.apnr.2004.05.002

[15] Resnicow, K.L., Baranowski, T., Ahluwalia, J.S. and Braithwaite, R.L. (1999) Cultural Sensitivity in Public Health: Defined and Demystified. Ethnicity \& Disease, 9, 10-21.

[16] US Department of Health and Human Services and US Department of Agriculture (2005) Dietary Guidelines for Americans. US Government Printing Office, Washington DC.

[17] US Department of Health and Human Services and US Department of Agriculture (2010) Dietary Guidelines for Americans. www.dietaryguidelines.gov

[18] US Department of Health and Human Services (2008) Physical Activity Guidelines for Americans. http://www.health.gov/paguidelines

[19] Krueger, R.A. (2000) Focus Groups: A Practical Guide for Applied Research. Sage Publications, California.

[20] National Institutes of Health (NIH), National Heart, Lung, and Blood Institute (2009) Ways to Enhance Children's Activity and Nutrition (We Can!). Program Brochure. www.nhlbi.nih.gov/health/public/heart/obesity/wecan

[21] Guidry, J.J., Fagan, P. and Walker, V. (1998) Cultural Sensitivity and Readability of Breast and Prostate Cancer Education Materials Targeting African Americans. Journal of the National Medical Association, 90, 165-169.

[22] McGee, B.B., et al. (2008) Perceptions of Factors Influencing Healthful Food Consumption Behavior in the Lower Mississippi Delta. Journal of Nutrition Education and Behavior, 40, 102-109. http://dx.doi.org/10.1016/j.jneb.2006.12.013

[23] Thomas, V.G. (1994) Using Feminist and Social Structural Analysis to Focus on the Health of Poor Women. Women Health, 22, 1-15. http://dx.doi.org/10.1300/J013v22n01_01

[24] Blixen, C.E., Singh, A. and Thacker, H. (2006) Values and Beliefs about Obesity and Weight Reduction among African American and Caucasian Women. Journal of Transcultural Nursing, 17, 290-297. http://dx.doi.org/10.1177/1043659606288375

[25] Parham, G.P. and Scarinci, I.C. (2007) Strategies for Achieving Healthy Energy Balance among African Americans in the Mississippi Delta. Preventing of Chronic Disease, 4, A97. http://www.cdc.gov/pcd/issues/2007/oct/07.0076.htm

[26] Duncan, D.T., Wolin, K.Y., Scharoun-Lee, M., Ding, E.L., Warner E.T. and Bennett, G.G. (2011) Does Perception Equal Reality? Weight Misperception in Relation to Weight-Related Attitudes and Behaviors among Overweight and Obese US Adults. International Journal of Behavioral Nutrition and Physical Activity, 22, 8-20.

[27] Stevens, J., Kumanyika, S.K. and Keil, J.E. (1994) Attitudes toward Body Size and Dieting: Differences between Elderly Black and White Women. American Journal of Public Health, 84, 1322-1325. http://dx.doi.org/10.2105/AJPH.84.8.1322

[28] Boyington, J. E., Carter-Edwards, L., Piehl, M., Hutson, J., Langdon, D. and McManus, S. (2008) Cultural Attitudes toward Weight, Diet, and Physical Activity among Overweight African American Girls. Preventing of Chronic Disease, 5, A36. http://www.cdc.gov/pcd/issues/2008/apr/07_0056.htm

[29] Anderson, R.E., Crespo, C.J., Bartlett, S.J., Cheskin, L.J. and Pratt, M. (1998) Relationship of Physical Activity and Television Watching with Body Weight and Level of Fatness among Children: Results from the Third National Health and Nutrition Examination Survey. Journal of the American Medical Association, 279, 938-942. http://dx.doi.org/10.1001/jama.279.12.938

[30] Keihner, A.J., Sugerman, S., Linares, A.M., Rider, C.D., Egelski, E., Mitchell, P.R., Hudes, M. and Biehl, M. (2013) Low-Income Californians with Access to Produce in Their Home, School, Work, and Community Environments Eat More Fruits and Vegetables. California Department of Public Health, Sacramento. http://www.cdph.ca.gov/programs/cpns/Documents/FVAccessResearchBriefFinal.pdf 
[31] Harris, E. and Bronner, Y. (2001) Food Counts in the African American Community: Chartbook. Morgan State University, Baltimore.

[32] Connell, C.L., Yadrick, M.K., Simpson, P., Gossett, J., McGee, B.B. and Bogle, M.L. (2007) Food Supply Adequacy in the Lower Mississippi Delta. Journal of Nutrition Education and Behavior, 39, 77-83. http://dx.doi.org/10.1016/j.jneb.2006.10.007

[33] McGee, B.B., Johnson, G.S., Yadrick, M.K., Richardson, V., Simpson, P.M., Gossett, J.M., Thornton, A., Johnson, C. and Bogle, M.L.(2011) Food Shopping Perceptions, Behaviors, and Ability to Purchase Healthful Food Items in the Lower Mississippi Delta. Journal of Nutrition Education and Behavior, 43, 339-348. http://dx.doi.org/10.1016/j.jneb.2010.10.007

[34] Lee, S.M. (2005) Physical Activity Among Minority Populations: What Health Promotion Practitioners Should Know -A Commentary. Health Promotion Practice, 6, 447-452. http://dx.doi.org/10.1177/1524839904263818

[35] Office of Minority Health (2011) Obesity and African Americans. Office of Minority Health, New York. http://minorityhealth.hhs.gov/templates/content.aspx?ID=6456

[36] Boylan, S., Louie, J.C.Y. and Gill, T.P. (2012) Consumer Response to Healthy Eating, Physical Activity and WeightRelated Recommendations: A Systematic Review. Obesity Reviews, 13, 606-617. http://dx.doi.org/10.1111/j.1467-789X.2012.00989.x

[37] US Centers for Disease Control and Prevention (2005) Guide to Community Preventive Services: The Community Guide. Atlanta (GA). http://www.thecommunityguide.org/index.html/

[38] Pasick, R. (1997) Socioeconomic and Cultural Factors in the Development and Use of Theory. In: Glanz, K., Lewis, F.M. and Rimer, B.K., Eds, Health Behavior and Health Education: Theory, Research, and Practice, 2nd ed, Josey-Bass, San Francisco.

[39] Pasick, R.J., D'Onofrio, C.N. and Otero-Sabogal, R. (1996) Similarities and Differences across Cultures: Questions to Inform a Third Generation for Health Promotion Research. Health Education Quarterly, 23, S142-S161.

[40] Airhihenbuwa, C.O., Kumanyika, S., Agurs, T.D., Lowe, A., Saunders, D. and Morssink, C.B. (1996) Cultural Aspects of African American Eating Patterns. Ethn Health, 1, 245-260. http://dx.doi.org/10.1080/13557858.1996.9961793

[41] Champagne, C.M., Bogle, M.L., McGee, B,B,, Yadrick, K., Allen, H.R., Kramer, T.R., Simpson, P., Gossett, J., Weber, J. and Lower Mississippi Delta Nutrition Intervention Research Initiative (2004) Dietary Intake in the Lower Mississippi Delta Region: Results from the Foods of Our Delta Study. Journal of the American Dietetic Association, 104, 199-207. http://dx.doi.org/10.1016/j.jada.2003.11.011

[42] James, D.C. (2004) Factors Influencing Food Choices, Dietary Intake, and Nutrition-Related Attitudes among African Americans: Application of a Culturally Sensitive Mode. Ethn Health, 9, 349-367. http://dx.doi.org/10.1080/1355785042000285375

[43] Smith, S.M.K., Patricia, M., Guenther, P.M., Subar, A.F., Kirkpatrick, S.L. and Dodd, K.W. (2010) Americans Do Not Meet Federal Dietary Recommendations. Journal of Nutrition, 140, 1832-1838. http://dx.doi.org/10.3945/jn.110.124826

[44] National Center for Cultural Competence (2003) A Guide to Choosing and Adapting Culturally and Linguistically Competent Health Promotion Materials. http://nccc.georgetown.edu/documents/Materials_Guide

[45] Emily, B., Kahn, E.B., Ramsey, L.T., Brownson, R.C., Heath, G.W., Howze, E.H., Powell, K.E., Stone, E.J., Rajab, M.W., Corso, P., and the Task Force on Community Preventive Services (2002) Effectiveness of Interventions to Increase Physical Activity: A Systematic Review. American Journal of Preventive Medicine, 22, 73-107.

[46] Yancey, A.K., Kumanyika, S.K., Ponce, N.A., McCarthy, W.J., Jonathan, E., Fielding, J.E., Leslie, J.P. and Akbar, J.(2004) Population-Based Interventions Engaging Communities of Color in Healthy Eating and Active Living: A Review. Preventing of Chronic Disease, 1, A09. http://www.ncbi.nlm.nih.gov/pmc/articles/PMC544532

[47] Robert Wood Johnson Foundation (2009) The Active Living Diversity Project. http://www.rwj.org/files/publications/other/PublicDiversityReport.pdf 